\title{
Comparison of Endoscopic-Assisted Adenoidectomy with Conventional Method
}

\author{
${ }^{1}$ Renuka A Bradoo, ${ }^{2}$ Rahul R Modi, ${ }^{3}$ Anagha A Joshi, ${ }^{4}$ Vikas Wahane \\ ${ }^{1}$ Professor and Head, Department of Otolaryngology, Head and Neck Surgery, LTM Medical College and General Hospital \\ Mumbai, Maharashtra, India \\ ${ }^{2}$ Assistant Professor, Department of Otolaryngology, Head and Neck Surgery, LTM Medical College and General Hospital \\ Mumbai, Maharashtra, India \\ ${ }^{3}$ Associate Professor, Department of Otolaryngology, Head and Neck Surgery, LTM Medical College and General Hospital \\ Mumbai, Maharashtra, India \\ ${ }^{4}$ Senior Resident, Department of Otolaryngology, Head and Neck Surgery, Ram Manohar Lohia Hospital, New Delhi, India
}

Correspondence: Rahul R Modi, Assistant Professor, Department of Otolaryngology, Head and Neck Surgery, LTM Medical College and General Hospital, Room No. 441, 4th Floor, College Building, Sion, Mumbai-400022, Maharashtra, India e-mail: dr.rrmodi@gmail.com

\section{ABSTRACT}

Objective: To evaluate and compare the efficacy of endoscopic-assisted (EA) adenoidectomy with the conventional adenoidectomy (CA) with an aim to reduce rates of residual adenoid tissue after adenoidectomy.

Methods: A prospective randomized study involving 32 patients in which 16 underwent EA and 16 underwent CA. The outcomes compared were residual adenoid tissue after 3 months of surgery, operative blood loss, operative time and complications.

Setting: A tertiary care teaching hospital.

Results: EA was significantly better in terms of residual tissue left behind as compared to CA ( $p<0.05)$, similar operative blood loss and operative time with no difference in complications.

Conclusion: EA makes for a safe and efficacious way to do an adenoidectomy which has a significant advantage over CA.

Keywords: Conventional adenoidectomy, Powered adenoidectomy, Curettage, Blood loss, Eustachian tube, Choana.

\section{INTRODUCTION}

Adenoidectomy is one of the most common worldwide performed otolaryngologic procedures. ${ }^{1}$ It is performed for various indications, such as adenoid hypertrophy, obstructive sleep apnea, recurrent otitis media and otitis media with effusion. It is performed either alone or in association with tonsillectomy or ventilation tube insertion.

Over the years, the technique has evolved and many different surgical options are available. Apart from the conventional curettage method, there are others like powered adenoidectomy, ${ }^{2}$ radiofrequency ablation ${ }^{3}$ and the electrocautery. ${ }^{4}$ However, the curettage method is still the most commonly followed all over the world. ${ }^{1}$ Recurrence rates have been reported between $8 \%$ and $40 \%$ in the earlier studies which have dropped to $<1 \%$ owing to newer techniques. ${ }^{5}$ However, increasing cost and expertise are the major reasons why some of the newer techniques are not that commonly in use.

We propose the use of the endoscope-assisted (EA) method over the conventional adenoidectomy (CA) method. A study was carried out to assess the efficacy and compare the amount of residual tissue after 3 months of surgery.

\section{PATIENTS AND METHODS}

The prospective randomized study was carried out at the Department of Otolaryngology, Lokmanya Tilak Municipal General Hospital, Mumbai, after approval from the institutional ethical review committee between August 2009 and August 2010. A total number of 32 patients participated in the study. These were randomly divided into two groups, thus 16 patients were operated using the conventional adenoidectomy procedure and 16 patients underwent endoscope-assisted adenoidectomy.

A detailed history was obtained, and a complete ear, nose and throat examination was done in all patients. Patients were selected on the basis of history of mouth breathing, snoring, drooling of saliva, adenoid facies and ear complaints. An X-ray nasopharynx lateral view soft tissue exposure was mainly done to confirm the diagnosis. Routine hematological investigations for anesthesia fitness were done in all the patients.

All the patients underwent surgery in the form of either endoscopic-assisted (EA) or conventional adenoidectomy (CA) under general anesthesia. 


\section{Operative Technique: Endoscopic-Assisted Adenoidectomy}

A $0^{\circ}$ endoscope was passed after nasal decongestion with $4 \%$ xylocaine and adrenaline solution. The nasal cavity and adenoids were inspected. A Doyens mouth gag was applied and a St Clair Thompsons adenoid curette was passed behind the soft palate. Using the endoscope, the curette was visualized in the nasopharynx and the adenoid tissue was curetted under vision (Figs 1 and 2). A conventional curettage adenoidectomy ${ }^{6}$ was performed in the remaining patients.

All the patients were followed up for a period of 3 months. History of symptomatic relief and resolution of middle ear effusion was noted. A postoperative nasal endoscopy was done after 3 months in all patients.

Residual adenoid tissue in the nasopharynx after adenoidectomy was divided into two grades for the purpose of comparison.

Grade I (minimal): Residual adenoid tissue was found above the level of the eustachian tube.

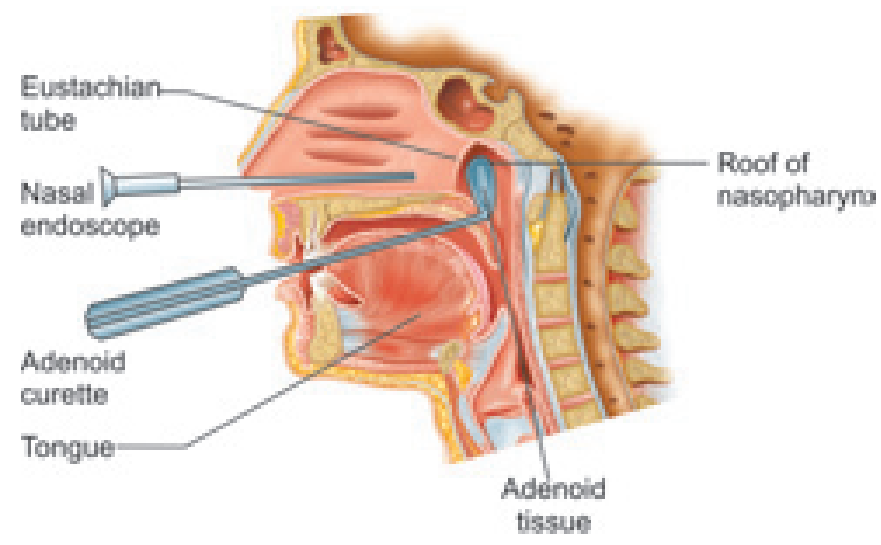

Fig. 1: Schematic representation of endoscopic-assisted adenoidectomy

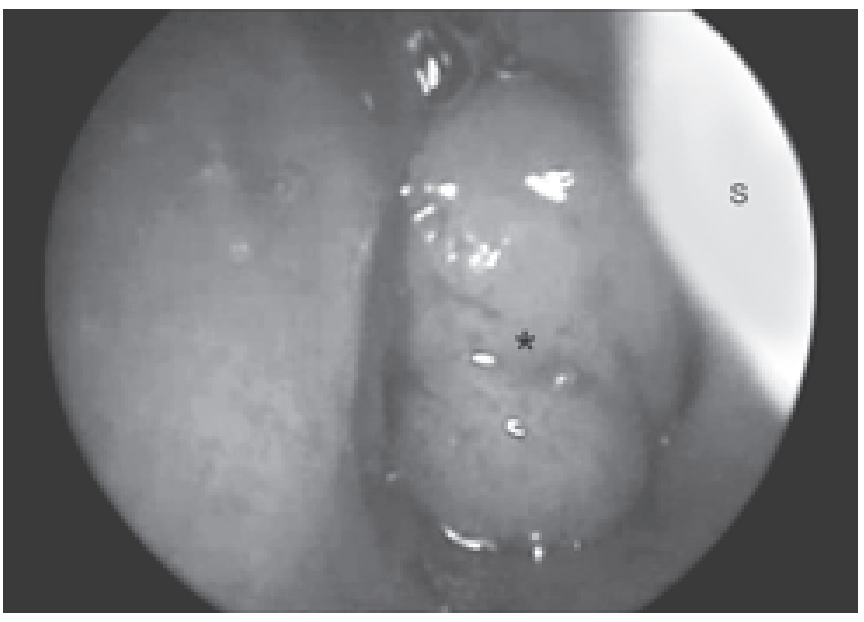

Fig. 2: Preoperative photo of adenoids $\left(^{*}\right)$ (S-septum)
Grade II: Residual adenoid tissue was found between the superior border of eustachian tube and a line extrapolated posteriorly from the floor of the nasal cavity. This grade was further subdivided into:

Grade IIa (moderate): Residual adenoid tissue obstructing the eustachian tube lumen but not blocking the posterior choana.

Grade IIb (severe): Residual adenoid tissue obstructing the eustachian tube lumen and blocking the posterior choana.

Various parameters, such as intraoperative blood loss, operative time and postoperative complications, were also compared.

\section{OBSERVATIONS AND RESULTS}

EA was performed in 16 patients ( 9 boys, 7 girls, mean age 8.2 years, age range $5-13$ years) and CA was performed in 16 patients ( 8 boys, 8 girls, mean age 8.3 years, range $5-13$ years) for a total of 32 patients. There was no statistical difference in terms of age and sex.

Results of the study were evaluated using the various parameters. There was a greater incidence of higher grade of residual adenoid tissue after 3 months of surgery in CA as compared to EA $(p<0.05)$ (Table 1, and Figs 3 and 4).

Table 1: Residual adenoid tissue after 3 months of surgery

\begin{tabular}{lll} 
Residual adenoid tissue & $\begin{array}{l}\text { Conventional } \\
\text { adenoidectomy } \\
(n=16)\end{array}$ & $\begin{array}{l}\text { Endoscopic- } \\
\text { assisted } \\
\text { adenoidectomy } \\
(n=16)\end{array}$ \\
\hline Grade I (minimal) & $5(31.2 \%)$ & $4(25 \%)$ \\
Grade IIa (moderate) & $7(43.7 \%)$ & $1(6.2 \%)$ \\
Grade IIb (severe) & $2(12.5 \%)$ & Nil \\
\hline Total & $14(87.5 \%)$ & $5(31.2 \%)$ \\
\hline
\end{tabular}

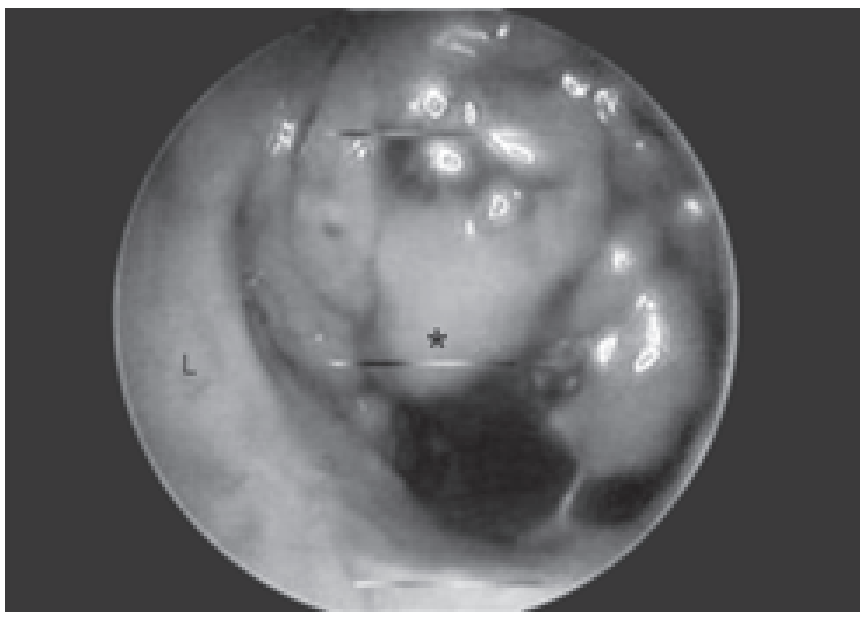

Fig. 3: Moderate residual tissue $\left({ }^{*}\right)$ postconventional adenoidectomy (L-lateral nasal wall) 


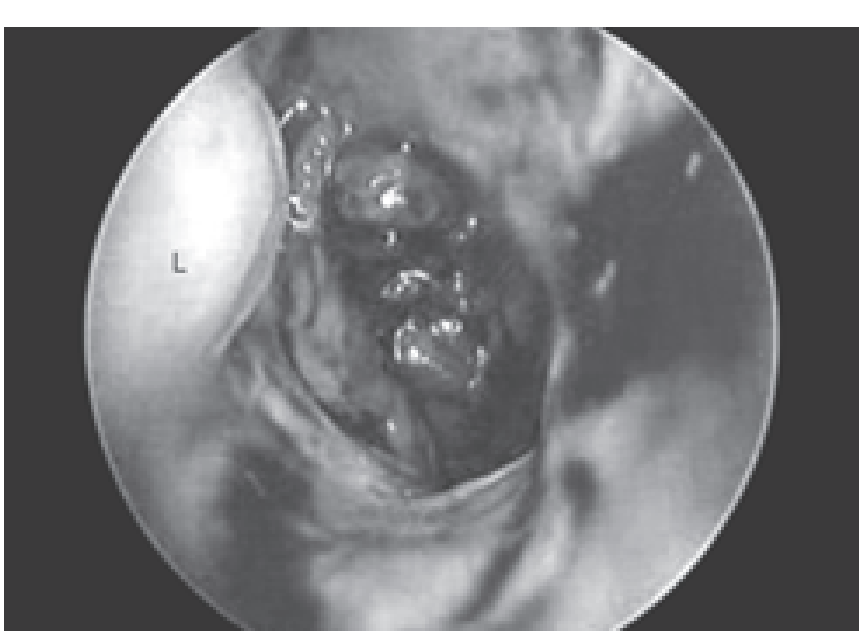

Fig. 4: Intraoperative photograph of endoscopic-assisted adenoidectomy (EA) showing near complete removal of adenoid tissue ( $L$-lateral nasal wall)

The mean blood loss in CA was $33 \mathrm{cc}$, and in EA, it was 38 cc $(p>0.05)$. The mean operative time in CA was 9 minutes while in EA it was 14 minutes $(\mathrm{p}<0.05)$.

No postoperative complications in the form of postoperative bleeding, velopharyngeal insufficiency, atlantoaxial dislocation, eustachian tube scarring, etc. were observed in either group.

\section{DISCUSSION}

Obstruction of the nasopharyngeal airway due to chronic adenoiditis is caused by the relative size of the adenoids to the size of the nasopharyngeal airway. The goal of the surgery is mainly to achieve a patent nasopharyngeal airway and relieve obstruction to the eustachian tube. The ideal approach should relieve the obstruction and leave minimal or no tissue in the nasopharynx and achieve good postoperative results.

Most techniques of adenoidectomy concentrate on removing predominantly the midline mass of adenoid tissue. The adenoid curette is used to remove tissue from the posterior edge of the vomer inferiorly to the level of superior constrictor muscle. Beneath this layer and behind the prevertebral fascia lie the cervical vertebrae. Their contour is rounded and does not match the perfectly straight edge of the instrument. Often, therefore, lateral lymphoid tissue remains in the nasopharynx, especially at the level of eustachian tube. That lymphoid tissue specifically projecting into the eustachian tube is known as the tonsil of Gerlach. An ideal adenoidectomy in young children should attain direct visualization, ease of procedure, short operating time, minimal blood loss, suitable cost, cure of symptoms and more importantly complete adenoid resection with no complications.
Clinical importance of minimal adenoid tissue being left behind may be low and may not lead to a symptomatic recurrence. However, following CA a large number (43.7\%) of patients had moderate residual disease which later might lead to recurrence of symptoms.

As with any new surgical technique, there is a learning curve to endoscopic-assisted adenoidectomy. Initially, the surgery appears to take more time but our results demonstrate a trend toward decrease in the time of the procedure with experience. The time required for preoperative nasal decongestion was additional to that in CA. Using the endoscope-assisted technique, the adenoid tissue removal was complete and more often to the appropriate depth, neither too shallow (leaving residual adenoid tissue) nor too deep (causing damage to surrounding structures).

Another similar technique is described by Ezzat ${ }^{5}$, whereby the residual adenoid tissue is removed by using an endoscope after conventional curettage. However, this technique makes use of a microdebrider to remove residual adenoid tissue adding to the cost of the surgery.

Many centers have adopted the method of powered endoscopic adenoidectomy with a microdebrider. It has the advantage of removing adenoids under vision thus confirming adequate adenoid tissue removal and avoiding damage to eustachian tube area. However, these prove to be expensive and require special training.

Our results show that intraoperative blood loss for surgery is similar in both methods. The blood loss during EA $(38 \mathrm{ml})$ was even similar to that during powered adenoidectomy $(35 \mathrm{ml}){ }^{2}$ Both groups also showed improvement in symptoms and the rate of complications was not different in either group. However, the two methods differed significantly in the amount of adenoid tissue left behind. Although, this may not have immediate clinical implications, there is a definite possibility of regrowth of the residual adenoid tissue causing a recurrence of nasal and ear symptoms, thus requiring revision surgery. ${ }^{2}$

The reason for incomplete removal of adenoids in the region of the roof of the nasopharynx and posterior choana is the inability of the curette to reach these areas easily. Residual adenoid tissue in the peritubal area may be due to apprehension on the part of the operating surgeon of damage to the tubal orifice and subsequent scarring.

\section{CONCLUSION}

Thus, endoscopic-assisted adenoidectomy is a safe and effective method of adenoidectomy. Visualization with an endoscope is helpful in achieving a complete adenoidectomy 
and preventing complications. Blind adenoid curettage was associated with higher residual adenoid tissue in a large number of cases.

\section{REFERENCES}

1. Van Den Akker EH, Hoes AW, Burton MJ, Schilder AGM. Large international differences in (adeno)tonsillectomy rates. Clinical Otolaryngology and Allied Sciences 2004;29(2):161-64.

2. Murray N, Fitzpatrick P, Guarisco JL. Powered partial adenoidectomy. Arch Otolaryngol Head Neck Surg July 2002; 128(7):792-96.
3. Timms MS, Ghosh S, Roper A. Adenoidectomy with the coblator: A logical extension of radiofrequency tonsillectomy. J Laryngol Otol May 2005;119(5):398-99.

4. Elluru RG, Johnson L, Myer CM 3rd. Electrocautery adenoidectomy compared with curettage and power-assisted methods. Laryngoscope Aug 2002;112(8 Pt 2 Suppl 100): 23-25.

5. Ezzat WF. Role of endoscopic nasal examination in reduction of nasopharyngeal adenoid recurrence rates. Int J Pediatr Otorhinolaryngol Apr; 74(4):404-06.

6. Kornblut AD. A traditional approach to surgery of the tonsils and adenoids. Otolaryngol Clin North Am May 1987;20(2):349-63. 